\title{
Old computers may prove best
}

Tokyo

Just at the time IBM (International Business Machines) drastically scaled down its research and development of Josephson junctions, Professor Eichi Goto of Tokyo University plunged into investigation of the supercooled logic devices, an idea originally developed by him more than 30 years ago. Now, three years later, Goto has been awarded about Y15,000 million ( $\$ 92$ million) by Japan's Research and Development Corporation under the ERATO programme to develop a Josephson junction computer that will process information in the form of minute magnetic fluxes.

The 'quantum magnetic flux logic' proIndian resources

\section{National water policy at last}

\section{Bangalore}

INDIA has been trying for many years without success to evolve a comprehensive national water policy, but this goal is now a step nearer.

The chief need is for efficient and integrated management of water resources and more effective use of irrigation facilities. Mr B. Shankarananda, Minister for Water Resource Management, says the new policy, which is now getting its final touches, is intended to make available safe drinking water for more than half a million villages and also to link the Himalayan and peninsular rivers so that surplus water can be transferred to areas that need it. Cost-effective techniques for finding water in drought-prone areas, particularly in the north-eastern part of the country, are also being sought.

Under the new policy, both central and state governments would share the cost of creating water channels, dams and drinking-water sources. Water would be declared a national asset so as to end interstate disputes.

In the meantime, the Indian Planning Commission has announced that irrigation and flood control projects that do not include plans for 'ancillary aspects' such as soil conservation and protection of the ecosystem will not be permitted.

Water-borne diseases account for the loss of 73 million man-days in India each year. In 1980, it was estimated that out of 570,000 Indian villages, more than a quarter have no source of drinking water within $16 \mathrm{~km}$. Although Rs 77 million had been recommended by a working group for rural water supply and sanitation in 1986-90, only Rs34.5 million was allocated.

Radhakrishna Rao ject will span five years and will be carried out by three research groups: Hitachi Central Research Laboratory will build the hardware, a team at Mitsui Shipbuilding will develop the software and a group at ULVAC in Chibasaki near Tokyo will be responsible for the magnetic shielding and refrigeration.

Goto achieved worldwide fame in the 1950 s with his patenting of the parametron, a resonant electrical circuit that can store binary information by utilizing the two possible phases of oscillation of the resonating current. But although several Japanese companies, including NEC and Hitachi, developed parametron computers, these machines were soon superseded by much faster transistorized computers in the $1960 \mathrm{~s}$, and parametron computers can now be found only in science museums.

Nevertheless, Goto believes that a Josephson junction computer using a new type of superconducting parametron could operate at $10 \mathrm{GHz}$, a hundred times faster than present-day computers, while consuming a thousandth of the power of other Josephson junction devices. The
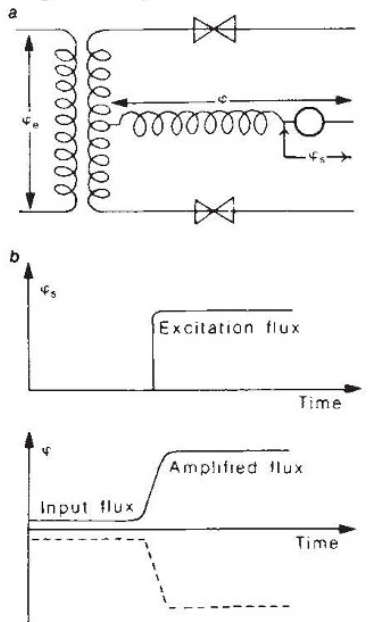

A d.c. flux parametron.

secret lies in using magnetic flux, rather than electrical current, as the conveyor of digital information.

The d.c. flux parametron outputs an amplified magnetic flux the polarity of which is determined by that of a small input signal (see figure). The two possible polarities, positive or negative, can represent the $0 \mathrm{~s}$ and $1 \mathrm{~s}$ of binary logic, and by linking parametrons together magnetically, all the basic logic circuits of a central processor can be built. And in fact Hitachi has already linked together three such parametrons to make a clocking device that operates at about $2 \mathrm{GHz}$.

One major problem, however, remains. The magnetic fluxes on which this computer is supposed to operate are so tiny, on the order of nanogauss, that the computer

\section{UK-Dutch telescope}

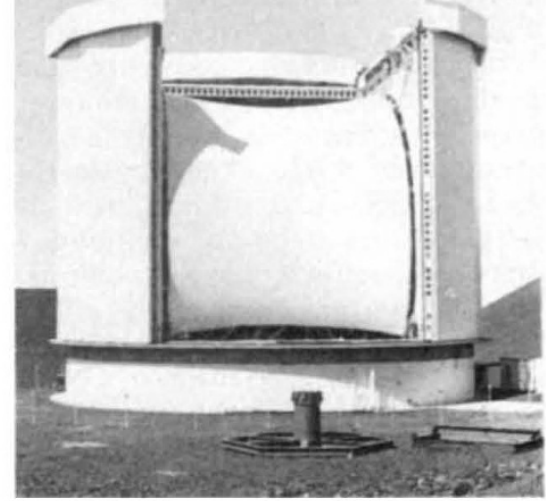

LAST week the James Clerk Maxwell Telescope, in the final stages of its initial commissioning tests, received its first radio sig-

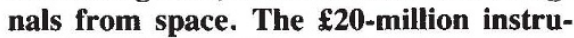
ment, installed at Mauna Kea Observatory on Hawaii, is the largest telescope in the world operating in the submillimetre region of the spectrum.

The telescope, jointly funded by Britain's Science and Engineering Research Council (SERC) and the Dutch Nederlandse Organisatie voor Zuiver-Wetenschappelijk Onderzoek (ZWO) will become fully operational next year.

The first radio signals to reach the telescope last Thursday were those from the Moon, Jupiter and Mars. The telescope has been specifically designed, however, to penetrate the dense regions of interstellar gas, which are opaque to optical radiation. At the infrared and millimetre wavelengths, these regions are transparent.

Bill Johnstone

must be completely shielded from external magnetic fields, such as that of the Earth and underground electric trains.

Undeterred, Goto has already set about trying to build the "perfect magnetic shield". In theory, a superconducting shield encasing the computer could shut out the external magnetic field. But in practice magnetic flux is pinned to the superconducter during cooling, setting up localized internal fields. Goto's plan is to 'sweep' the shield clean of pinned flux with a laser beam, but so far without success.

During preliminary experiments with a superconducting cylinder made of lead, more than 99.9 per cent of the laser's light was reflected by the shiny lead surface and a 'hot spot' for collecting flux failed to develop. Maintaining the cylinder black did not help much either - the paint peeled off on cooling.

If successful, Goto will be able not only to protect the computer from magnetic fields but will also have the ideal detector for magnetic monopoles, which were predicted by the late P.A.M. Dirac.

David Swinbanks 